\title{
Potential Energy Landscape of Monolayer-Surface Systems Governed by Repulsive Lateral Interactions: The Case of $(3 \times 3)$-I-Pt $(111)$
}

\author{
Alexandre Tkatchenko, ${ }^{*}$ Nikola Batina, and Marcelo Galván \\ Departamento de Química, División de Ciencias Básicas e Ingeniería, Universidad Autónoma Metropolitana-Iztapalapa, \\ San Rafael Atlixco 186, Vicentina, A.P. 55-534, México D.F. 09340, México
}

(Received 21 March 2006; published 18 July 2006)

\begin{abstract}
Combined density functional theory (DFT) and Monte Carlo (MC) approach is applied to study the potential energy landscape of four iodine atoms adsorbed on the $\operatorname{Pt}(111)$ surface in a $(3 \times 3)$ unit cell. Three critical points were identified: $(3 \times 3)$-sym and $(3 \times 3)$-asym, corresponding to structures well known from experimental studies, while the third one $(3 \times 3)$-zigzag is a new structure not reported before. An interaction model fitted to DFT calculations allows us to explain the difference between arrangements of iodine monolayer in vacuum, air, and solution environments as a result of different repulsion regimes.
\end{abstract}

DOI: 10.1103/PhysRevLett.97.036102

Investigation of potential energy landscapes of different systems, such as clusters, biomolecules, and glasses, have attracted much attention over the last years [1]. The availability of high quality interaction potentials makes possible detailed analysis of minima and saddle points in order to gain valuable insight into kinetics and dynamics of these systems. On the other hand, adsorbates on well-ordered surfaces represent a system where interaction potentials are available in very few cases, mainly for rare gases adsorbed on metal surfaces [2]. For many chemisorption systems, not even a phenomenological picture of interactions is available yet. Density functional theory (DFT) calculations allow a detailed description of the potential energy surface (PES) and nowadays are routinely applied for the description of the adsorbate-substrate systems $[3,4]$. The shortcoming of DFT is the time required to perform the calculations, restricting the sampling of the PES to about $10^{2}-10^{3}$ points even for adsorption systems with few atoms. Different methodologies have been proposed for constructing complete analytic PES based on a limited number of DFT energies [5-7], but they are restricted to systems with a small number of dimensions and still require considerable computational effort. On the experimental side, the wealth of data available for the structure of different adsorbate-substrate systems [8] makes the detailed theoretical study of their energy landscapes very interesting. In this Letter, we use combined DFT and Monte Carlo (MC) approach to analyze the PES of four atoms of iodine adsorbed on the $\operatorname{Pt}(111)$ surface in a $(3 \times$ 3 ) unit cell. We identify three extreme points on the PES. Among the three extremes, two of them correspond to experimentally known structures and one is a new nonreported structure for this system. From characterization of the Hessian matrix and MC simulations, one can conclude that the stabilization of different structures can be rationalized by two different repulsion regimes. High-repulsion regime corresponds to experimental results in air and
PACS numbers: 68.35. $-\mathrm{p}$, 68.43. $-\mathrm{h}, 71.15 . \mathrm{Mb}, 75.40 . \mathrm{Mg}$

solution environments, while low-repulsion reproduces vacuum results.

The I-Pt(111) system is one of the most well studied in the surface science and electrochemistry community [919]. From the experimental point of view, it is easy to prepare and maintain stability in vacuum, air, and solution environments, which was demonstrated in numerous studies so far [19]. I-Pt(111) shows four different commensurate structures: $(\sqrt{3} \times \sqrt{3}) R 30^{\circ},(\sqrt{7} \times \sqrt{7}) R 19.11^{\circ}$, $(3 \times 3)$-sym, and $(3 \times 3)$-asym. The $(3 \times 3)$ unit cell with four atoms is particularly interesting because of coexistence of two structures with different symmetry. Early low energy electron diffraction (LEED) studies of the I-Pt(111) system [9] found indication of the $(3 \times 3)$ structure. The sym and asym symmetries could not be distinguished, because their LEED patterns are essentially the same. Scanning tunneling microscopy (STM) experiments in air by Schardt et al. [10] distinguished between sym and asym structures and showed the topographic differences between the two. Several other STM studies in air and in solution confirmed the coexistence of both structures and found them in approximately the same concentration at the $\operatorname{Pt}(111)$ surface $[12,14,19]$. More recent tensor LEED study in vacuum [16] could only find evidence of the $(3 \times 3)$-sym structure at coverage $\theta=4 / 9$. The structure with $(3 \times 3)$-asym symmetry could not be fitted to experimental LEED spectra.

The DFT calculation procedure used in this work is based on a slab model that includes five layers of substrate Pt atoms with iodine atoms (monolayer) adsorbed on both sides of the slab. The electronic structure was calculated within the Kohn-Sham DFT formalism [20] using the generalized gradient approximation (GGA) with the PerdewBurke-Ernzerhof (PBE) functional form [21]. The KohnSham equations were solved using a plane-wave expansion truncated at $40 \mathrm{Ry}$ within the DFT ++ program [22]. The core electrons were not treated explicitly and norm con- 
serving Troullier-Martins type pseudopotentials [23] were employed. The use of $4 k$ points in the irreducible zone was determined as appropriate to converge the total energy up to one millihartree. Some additional tests with a larger $k$-point set did not modify our results. The adsorbates and the first two layers of platinum surface were fully relaxed with forces converged up to $10^{-3}$ hartree/bohr and stress on the unit cell was minimized. The optimized unit cell was of $8.48 \times 8.48 \times 27 \AA^{3}$. The level of theory described above provides excellent agreement with experimental results [24].

In order to map the stationary points of the $(3 \times 3)$-I$\mathrm{Pt}(111)$ system, we performed the relaxation of a systematic and uniform sample of the $(3 \times 3)$ unit cell within the hexagonal symmetry restriction (the cell parameters were always kept fixed). This set of structures has been recently analyzed in a geometrical study of the $(3 \times 3)-\mathrm{I}-\mathrm{Pt}(111)$ system [25]. The choice of starting points with hexagonal symmetry was motivated by experimental results where only hexagonal structures have been found. We identified three different extreme points, shown on Fig. 1, which we will call $(3 \times 3)$-sym, $(3 \times 3)$-asym, and $(3 \times 3)$-zigzag [26]. The first two structures are well known from different experimental studies $[10,12,14,16]$, while the third one has never been observed for I-Pt(111). Note that the sym and asym structures possess almost perfect hexagonal order inside the $(3 \times 3)$ unit cell, while the iodine atoms positioned close to fcc and hcp sites in the $(3 \times 3)$-zigzag structure relax around $0.3 \mathrm{~A}$ from the perfect hexagonal arrangement. In order to classify the extreme points, we analyzed the Hessian matrix eigenvalues, formed by numerical second derivative evaluation for the four iodine atoms in the unit cell, which showed that $(3 \times 3)$-sym and $(3 \times 3)$-zizgag are minima, while $(3 \times 3)$-asym is a saddle point. The adsorption energies of these structures and their geometrical parameters, as obtained from GGA calculations, are presented in Table I. The structural parameters show the expected trend [24], namely, that atoms in three-
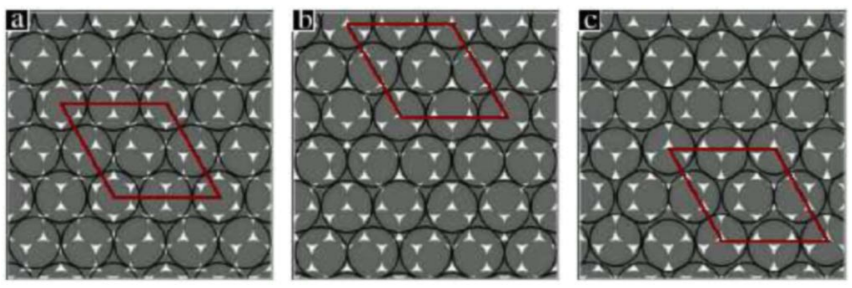

FIG. 1 (color online). Models of three structures in the $(3 \times 3)$ cell. The figure shows two layers; the black circles represent the iodine atoms and gray filled circles represent the first layer of $\mathrm{Pt}(111)$ atoms. The $(3 \times 3)$ unit cell is indicated by a red rhomb in the three cases. (a) $(3 \times 3)$-sym with one on-top iodine atom and three bridge-positioned iodine atoms. (b) $(3 \times 3)$-asym with one threefold fcc iodine atom and three iodine atoms with asymmetric registry. (c) $(3 \times 3)$-zigzag with one threefold fcc iodine atom, one threefold hcp atom, and two iodine atoms in asymmetric positions [different from $(3 \times 3)$-asym]. fold sites have the lowest equilibrium positions, with bridge and atop following. A simple reaction coordinate can be established between sym and asym structures. It is formed by collective movement of all four iodine atoms in the unit cell following a linear trajectory on the $X-Y$ plane with one bridge atom sliding into the closest fcc site. We have calculated the energy of ten different points along that trajectory by fixing the $X-Y$ position of the adsorbate atoms and allowing the vertical positions to be relaxed. We found that the adsorption energy decreases monotonically, thus confirming the fact that $(3 \times 3)$-asym is indeed a saddle point. Such simple reaction coordinate cannot be established for transitions between the structures with sym or asym and zigzag symmetry, due to the noticeable atomic relaxation inside the unit cell of the zigzag structure.

Considering that $(3 \times 3)$-sym and $(3 \times 3)$-asym are the unique structures observed in experiments $[10,12,14,16]$, at first glance it is surprising that $(3 \times 3)$-asym is a saddle point and $(3 \times 3)$-zizgag is a local minimum. Experimental studies in solution and air environments have found a mixture of $(3 \times 3)$-sym and $(3 \times 3)$-asym at the $\operatorname{Pt}(111)$ surface. In contrast, a recent study in vacuum [16] indicated that no evidence for $(3 \times 3)$-asym structure could be found. Our DFT results are closer to the vacuum experimental situation and this explains why the saddle point, $(3 \times 3)$-asym, has not been observed. The experimental study in vacuum did not analyze the possibility of formation of the $(3 \times 3)$-zizgag structure, because it has never been reported before.

In order to determine the reason for the stabilization of the observed structures in air, solution, and vacuum environments, we use a simple energy expression. Following many other adsorption studies [2,4], we separate the I$\mathrm{Pt}(111)$ interaction potential into adsorbate-substrate and adsorbate-adsorbate parts:

$$
E=\sum_{i}^{N}\left\{V\left(x_{i}, y_{i}\right)+\sum_{j>i}\left[A \exp \left(-B r_{i j}\right)\right]\right\}
$$

where $r_{i j}$ is the distance between particles $i$ and $j$, while $A$ and $B$ are parameters. $N$ is equal to 4 for $(3 \times 3)$ cell with coverage $\theta=4 / 9$. The second summation is cut after $15 \AA$ A Tests with larger cutoff did not affect our results.

We assume a simple exponential interaction between the iodine atoms in the adsorbed monolayer. Although, clearly a simplification, several experimental studies point out that the interaction between adsorbed iodine atoms is repulsive in a wide range of coverages [18]. Furthermore, the $(3 \times 3)$ cell with coverage $\theta=4 / 9$ is the most compressed one observed in experiments. In order to set limits on the $A$ and $B$ parameters, we performed calculations of iodine adsorbed on the fcc site in $(\sqrt{3} \times \sqrt{3}) R 30^{\circ}$ cell with $\theta=\frac{1}{3}$ and in the $(1 \times 1)$ cell with $\theta=1$. We use the adsorption energy of the $\sqrt{3}$ structure to fix the $A$ parameter, while the variation of $B$ parameter allows us to simulate the effect of repulsion between iodine atoms in the monolayer. The 
TABLE I. GGA adsorption energies (Ad.) and $X, Y, Z$ structural parameters of four iodine atoms for the three structures of the $(3 \times 3)-\mathrm{I}-\mathrm{Pt}(111)$ system. The energies are reported in $\mathrm{kcal} / \mathrm{mol}, X-Y$ structural parameters in reduced unit cell coordinates, and $Z$ positions in $\AA$ measured relative to the first Pt(111) surface layer.

\begin{tabular}{lcclrc}
\hline \hline & Ad. & \multicolumn{1}{c}{$I_{1}(X, Y, Z)$} & \multicolumn{1}{c}{$I_{2}(X, Y, Z)$} & $I_{3}(X, Y, Z)$ & $I_{4}(X, Y, Z)$ \\
\hline$(3 \times 3)$-sym & 217.9 & $(-0.001,-0.001,2.774)$ & $(0.503,-0.007,2.255)$ & $(-0.005,0.503,2.276)$ & $(0.504,0.505,2.248)$ \\
$(3 \times 3)$-asym & 210.0 & $(-0.112,0.222,2.091)$ & $(0.389,0.220,2.499)$ & $(-0.117,0.725,2.483)$ & $(0.391,0.726,2.490)$ \\
$(3 \times 3)$-zigzag & 212.2 & $(0.078,0.002,2.612)$ & $(0.587,-0.002,2.612)$ & $(0.099,0.472,2.204)$ & $(0.564,0.534,2.129)$ \\
\hline \hline
\end{tabular}

following values were used for $A(\mathrm{kcal} / \mathrm{mol})$ and $B$ (reduced unit cell coordinates ${ }^{-1}$ ): $A=8 \times 10^{5}$ and $4.5 \leq$ $B \leq 5$. Smaller value of $B$ means higher repulsion. We will show that the manipulation of this single parameter is enough to fulfill the purpose of our study.

The adsorbate-substrate interaction $V(x, y)$ for the iodine atom with the $\operatorname{Pt}(111)$ surface is represented by the unequal-sphere packing (USP) potential, which has been shown to give an astonishing agreement with experimental structures of the I-Pt(111) system [25,27].

$$
V(x, y)=k\left[\sqrt{\left(R_{1}+R_{2}\right)^{2}-\left(x-x_{s}\right)^{2}-\left(y-y_{s}\right)^{2}}\right]+b .
$$

The quantity between the parenthesis is the equilibrium $Z$ distance of an iodine atom measured relative to the first $\mathrm{Pt}$ surface layer, $R_{1}$ is the surface atoms metallic radius, $R_{2}$ is the adsorbate radius and $\left(x_{s}, y_{s}\right)$ is the closest substrate particle position. $k$ and $b$ are the parameters to be fitted to $a b$ initio data. Note that $R_{2}$ is set to represent the equilibrium bond lengths of the adsorbate atoms. The $b$ parameter is only needed for absolute energy values. Therefore, $k$ is the only parameter which needs to be fitted for the adsorbate-substrate interaction potential. It is related to the corrugation of the potential. We fit the parameters of USP potential with the adsorption energies and equilibrium $Z$ positions of fcc and atop sites from our previous DFT study [24]. The values of parameters of the USP model used here are $R_{1}=1.3875 \AA, R_{2}=1.8122 \AA, k=$ $-22.8140 \mathrm{kcal} / \mathrm{mol}, b=96.9091 \mathrm{kcal} / \mathrm{mol}$. In Fig. 2, the excellent performance of the USP potential in comparison to DFT calculations is shown (see Fig. 2 caption for more details). The linear relation between $E$ and $Z$ obtained in DFT calculations is striking and it would be interesting to look for such behavior in other adsorbatesubstrate systems.

According to Eq. (1), once we define the parameters for $V(x, y)$ and the constant $A$, the global interaction is a direct function of a single parameter $-B$, which defines different repulsion regimes and induces deformation of the PES as it will be shown below. We have performed Monte Carlo (MC) simulations with the interaction potential of Eq. (1), exploring the PES of the $(3 \times 3)-\mathrm{I}-\mathrm{Pt}(111)$ system containing four iodine atoms per unit cell. Periodic boundary conditions have been used for the $X$ and $Y$ directions of the unit cell and the MC simulation procedure was as follows: $100 \mathrm{MC}$ runs (without Boltzmann factor) were performed for each value of $B$ in the interval $4.5 \leq B \leq 5$ with increments of 0.01 . We did additional runs around $B=4.74$ in order to verify that all three structures do not coexist for any value of $B$. Each MC run consisted of the following steps: (1) initializing randomly the $X-Y$ positions of all four atoms in the unit cell, (2) randomly moving one of the four atoms within a circle of radius 0.001 reduced unit cell coordinates, (3) the step is accepted if the energy of new configuration is lower than the previous one, (4) repeating steps (2)-(3) until reaching convergence. The final configuration was accepted if the energy did not change more than $10^{-4}$ in $5000 \mathrm{MC}$ steps. For $B<4.74$, $(3 \times 3)$ sym and $(3 \times 3)$-asym were the only minima, while for $B \geq 4.74,(3 \times 3)$-sym and $(3 \times 3)$-zizgag were obtained exclusively. We could not find any value of $B$ around 4.74 , which showed the existence of the three structures as minima. Therefore, one could conclude that the deformation of the PES around $B=4.74$ is abrupt, with asym structure most probably becoming saddle point and zigzag

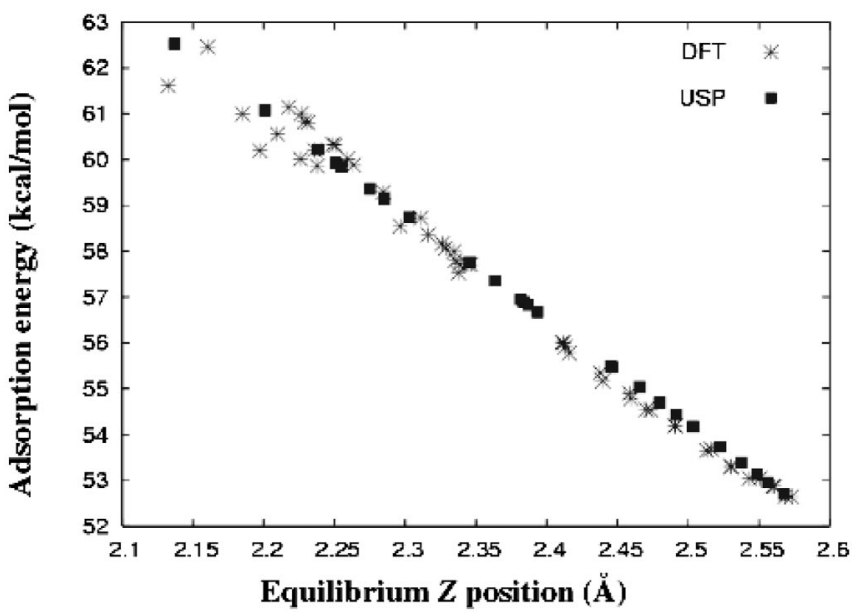

FIG. 2. Adsorption energy vs equilibrium $Z$ position for 50 different sites for one iodine atom in the $(3 \times 3)-\operatorname{Pt}(111)$ cell. The equilibrium position is measured relative to the first Pt layer. DFT calculations were performed with a three layer Pt(111) slab with iodine adsorbed on one side. The USP potential fitted to equilibrium $Z$ positions and adsorption energies of fcc and atop sites is in perfect agreement with DFT calculations. Note the almost perfect linear $E(Z)$ dependence, which only breaks around fcc-hcp sites, which have been shown to be degenerate for an extended model [see Ref. [24]]. 
becoming minimum with an increase of $B$. Note that higher values of $B$ imply lower repulsion. Summarizing, $(3 \times 3)$ sym is a global minimum for the interval of $B$ values in this study, while $(3 \times 3)$-asym is minimum for high repulsion and $(3 \times 3)$-zigzag is minimum for lower repulsion.

Making a comparison of our MC results with the abovementioned experimental data and DFT calculations, a simple picture emerges. It would seem that a high-repulsion regime corresponds to experimental studies in air and solution environments, while low repulsion corresponds to DFT results obtained in this study, which could be associated to vacuum. The existence of the structure with $(3 \times 3)$-zigzag symmetry is an intriguing possibility. Along this vein it is important to notice that the $(3 \times \sqrt{3} / 2)$-rect structure found by Orts et al. [28] in their experimental study of $\mathrm{Br}-\mathrm{Pt}(111)$ system is essentially the same as $(3 \times 3)$-zigzag. The fact that zigzag structure has been observed for $\mathrm{Br}-\mathrm{Pt}(111)$ could be rationalized by the smaller van der Waals radius of bromine atoms, which could indicate smaller effective repulsion between them in the adsorbed monolayer.

It is noticeable that a simple model, as the one proposed in this work, could explain the vast amount of experimental results in $(3 \times 3)-\mathrm{I}-\mathrm{Pt}(111)$ system. The origin of the higher repulsive regime in solution can be traced back to an induced charge transfer as has been observed in other halogen-metal adsorption systems, as could be seen, e.g., in Fig. 2 of Ref. [19] and the related discussion. This effect can be related with a dipolar repulsive interaction and has been used for an appropriate description in Monte Carlo simulations [29]. Such effect is not present in vacuum because the charge transfer is quite small [24]. In the presence of air the repulsion could be related to a threebody effect that modifies the I-I interaction. In order to test possible effect of the air environment, we have performed DFT calculations of an isolated iodine monolayer with $\mathrm{N}_{2}$ molecule on top and on the bridge positions. When the $\mathrm{N}_{2}$ molecule is positioned $2.5-3.5 \AA$ away from the monolayer, visible repulsion effect can be noticed in the monolayer interaction curve, which is increased when the molecule is closer.

In conclusion, we have performed combined DFT and MC study of the potential energy landscape of iodine adsorbed on the $\operatorname{Pt}(111)$ surface in $(3 \times 3)$ unit cell. Such an approach turned out to be powerful enough to rationalize differences between I-Pt(111) system in solution, air, and vacuum environments. We have found a new structure called $(3 \times 3)$-zigzag and we believe it is worth to pursue additional experimental studies of the I-Pt(111) system to confirm its existence.

All calculations were performed at Laboratorio de Supercomputo y Visualizacion en Paralelo at UAMIztapalapa and GRAMA facilities. A. T. acknowledges the organizers of the program Bridging Time and Length Scales in Materials Science and Biophysics which was held at IPAM/UCLA and CONACYT for financial support.
*Electronic address: sanix@ixil.izt.uam.mx

[1] D. J. Wales, Energy Landscapes: Applications to Clusters, Biomolecules and Glasses (Cambridge University Press, Cambridge, England, 2003).

[2] L.W. Bruch, M.W. Cole, and E. Zaremba, Physical Adsorption: Forces and Phenomena (Oxford University Press, New York, 1997).

[3] K. Horn and M. Scheffler, Handbook of Surface Science: Electronic Structure (North-Holland, Amsterdam, 2000).

[4] R. I. Masel, Principles of Adsorption and Reaction on Solid Surfaces (Wiley, New York, 1996).

[5] A. Groß and M. Scheffler, Phys. Rev. B 57, 2493 (1998).

[6] G. J. Kroes, Prog. Surf. Sci. 60, 1 (1999).

[7] L. Soenke, Ph.D. thesis, Fritz-Haber Institute, Berlin, 2001.

[8] G. A. Somorjai, Introduction to Surface Chemistry and Catalysis (Wiley, New York, 1994).

[9] A. T. Hubbard, Chem. Rev. 88, 633 (1988).

[10] B. C. Schardt, S. L. Yau, and F. Rinaldi, Science 243, 1050 (1989).

[11] S. L. Yau, C. M. Vitus, and B. C. Schardt, J. Am. Chem. Soc. 112, 3677 (1990).

[12] S. C. Chang, S. L. Yau, B. C. Schardt, and M. J. Weaver, J. Phys. Chem. 95, 4787 (1991).

[13] D. G. Frank, O. M. R. Chyan, T. Golden, and A. T. Hubbard, J. Phys. Chem. 97, 3829 (1993).

[14] J. Inukai et al., J. Phys. Chem. B 102, 3498 (1998).

[15] C. A. Lucas, N. M. Marković, and P. N. Ross, Phys. Rev. B 55, 7964 (1997).

[16] M. Saidy et al., Surf. Rev. Lett. 6, 871 (1999).

[17] J. H. Chen, S. L. Yau, and S. C. Chang, J. Phys. Chem. B 106, 9079 (2002).

[18] M. Labayen, S. A. Furman, and D. A. Harrington, Surf. Sci. 525, 149 (2003).

[19] O. M. Magnussen, Chem. Rev. 102, 679 (2002).

[20] R. G. Parr and W. Yang, Density Functional Theory of Atoms and Molecules (Oxford University Press, New York, 1989).

[21] J. P. Perdew, K. Burke, and M. Ernzerhof, Phys. Rev. Lett. 77, 3865 (1996).

[22] S. Ismail-Beigi and T. Arias, Comput. Phys. Comm. 128, 1 (2000).

[23] N. Troullier and J.L. Martins, Phys. Rev. B 43, 1993 (1991).

[24] A. Tkatchenko, N. Batina, A. Cedillo, and M. Galvan, Surf. Sci. 581, 58 (2005).

[25] A. Tkatchenko and N. Batina, J. Chem. Phys. 122, 094705 (2005).

[26] In Fig. 1(c) one can see that if the atoms on fcc and hcp sites are connected, a zigzag pattern emerges. One could also see that zigzag structure is characterized by the largest atomic relaxation inside the unit cell compared to the sym and asym structures.

[27] A. Tkatchenko and N. Batina, Phys. Rev. B 70, 195403 (2004).

[28] J. M. Orts et al., J. Phys. Chem. 100, 2334 (1996).

[29] I. Abou-Hamad, S. J. Mitchell, T. Wandlowski, P. A. Rikvold, and G. Brown, Electrochim. Acta 50, 5518 (2005). 